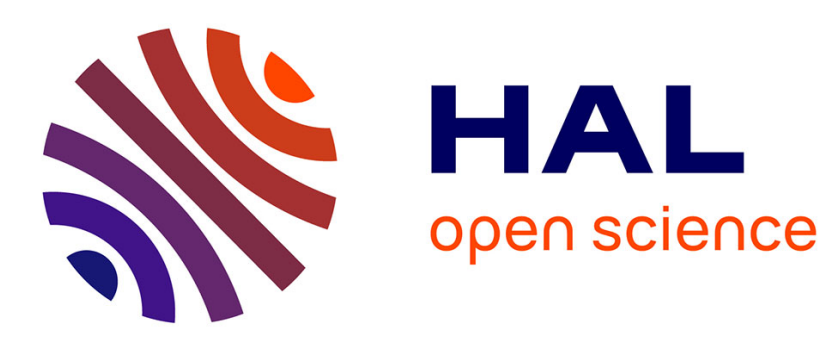

\title{
Étalonnage d'un spectrographe magnétique de type Buechner et reproductibilité des mesures
}

\author{
L. Bianchi
}

\section{To cite this version:}

L. Bianchi. Étalonnage d'un spectrographe magnétique de type Buechner et reproductibilité des mesures. Revue de Physique Appliquée, 1969, 4 (2), pp.211-212. 10.1051/rphysap:0196900402021100 . jpa-00243223

\section{HAL Id: jpa-00243223 https://hal.science/jpa-00243223}

Submitted on 1 Jan 1969

HAL is a multi-disciplinary open access archive for the deposit and dissemination of scientific research documents, whether they are published or not. The documents may come from teaching and research institutions in France or abroad, or from public or private research centers.
L'archive ouverte pluridisciplinaire HAL, est destinée au dépôt et à la diffusion de documents scientifiques de niveau recherche, publiés ou non, émanant des établissements d'enseignement et de recherche français ou étrangers, des laboratoires publics ou privés. 


\title{
ÉTALONNAGE D'UN SPEGTROGRAPHE MAGNÉTIQUE DE TYPE BUEGHNER ET REPRODUGTIBILITÉ DES MESURES
}

\author{
L. BIANCHI, \\ Service de Physique Nucléaire à Basse Énergie, G.E.N., Saclay.
}

\begin{abstract}
Résumé. - Les essais de reproductibilité et l'étalonnage ont été réalisés en utilisant les groupes ${ }^{4} \mathrm{He}^{++}$et ${ }^{4} \mathrm{He}^{+}$de ${ }^{122} \mathrm{Po}$ et ${ }^{212} \mathrm{Bi}$. La précision obtenue pour l'énergie est $\Delta E / E \leqslant 2 \times 10^{-4}$ pour un domaine de rigidité magnétique tel que $350<(B \rho)<850 \mathrm{kG}$.cm.
\end{abstract}

Abstract. - The reproductibility and calibration were performed using ${ }^{4} \mathrm{He}^{++}$and ${ }^{4} \mathrm{He}^{+}$from ${ }^{212} \mathrm{Po},{ }^{212} \mathrm{Bi}$. The precision obtained for the energy is : $\Delta E / E \leqslant 2 \times 10^{-4}$, for the following magnetic rigidity range $350<(B \rho)<850 \mathrm{kG} . \mathrm{cm}$.

I. La reproductibilité. - Au cours des expériences d'étalonnage de l'appareil, nous avons remarqué un déplacement relatif d'un pic donné suivant que l'induction de travail était obtenue par valeurs croissantes à partir de $B=0$ ou par valeurs décroissantes à partir de $B=B_{\max }$. Ce phénomène semble lié à une déformation non élastique des pièces polaires lors des variations d'induction. Nous avons alors recherché un « historique » de l'induction conduisant à une reproductibilité de la position des pics compatible avec la précision de l'appareil. Un détecteur à jonctions, muni d'une fente fine, a été placé en un point de la focale. Les raies $\alpha_{0}$ et $\alpha_{1}$ de ${ }^{212} \mathrm{Bi}$ ont été observées par spectrométrie, c'est-à-dire par variations lentes de l'induction, et pour différentes conditions d'origine de cette induction. Une reproductibilité meilleure que $1 \times 10^{-5}$ (pour $\Delta B / B$ ) a été trouvée dans les conditions suivantes : pour obtenir une induction de travail $B_{\mathrm{T}}$, il faut augmenter d'abord l'induction jusqu'à $B=B_{\max }$, la maintenir à cette valeur pendant cinq minutes, puis la faire décroître jusqu'à la valeur $B_{\mathrm{T}}$ (un deuxième cycle $B_{\mathrm{T}} \rightarrow B_{\mathrm{T}}$ est conseillé).

La valeur $B_{\mathrm{T}}$ ayant été obtenue de la façon précitée, nous avons pu vérifier, toujours par spectrométrie, qu'elle restait stable sur une période de 20 heures, à la précision de la mesure près. Enfin, des vérifications sur plaques nucléaires ont montré que, dans ces conditions, et pour une induction $B_{\mathrm{T}}$ donnée, les déplacements de raies $\alpha$ de ${ }^{212}$ Po et de ${ }^{212} \mathrm{Bi}$ sont restés inférieurs à $100 \mu \mathrm{m}$ lors d'expériences différentes effectuées à plusieurs jours d'intervalle.

II. L'étalonnage en énergie. - Il a été réalisé à l'aide des groupes ${ }^{4} \mathrm{He}^{++}$et ${ }^{4} \mathrm{He}^{+}$issus de sources de ${ }^{212} \mathrm{Bi},{ }^{212} \mathrm{Po}$; il couvre ainsi des valeurs de rigidité magnétique comprises entre 354 et 854 kG.cm (soit, en termes d'énergie de protons, de 6 à $35 \mathrm{MeV}$ ).
Chacun de ces groupes a été focalisé en différents points de la focale par variation systématique de l'induction. A partir de leurs rigidités magnétiques, nous avons calculé les valeurs d'induction qui les amèneraient à des abscisses théoriques $\left(S_{\mathrm{Th}}\right)$ prédéterminées. Le dépouillement des plaques nous a donné les abscisses mesurées $\left(S_{\mathrm{M}}\right)$ de ces groupes et les courbes de la figure $1 \Delta S=S_{\mathrm{M}}-S_{\mathrm{Th}}=f\left(S_{\mathrm{Th}}\right)$ nous ont servi de base pour le calcul des corrections à apporter.

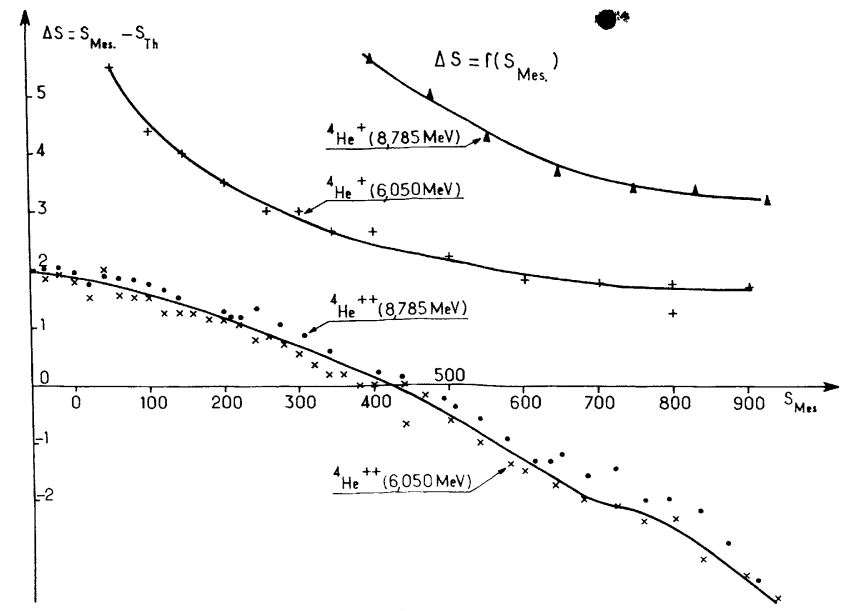

FIG. 1.

On peut remarquer ( fig. 1) que $\Delta S$ est sensiblement le même pour les groupes ${ }^{4} \mathrm{He}^{++}$de 6,05 et $8,78 \mathrm{MeV}$. De plus, cette variation est assez linéaire et nous avons supposé qu'il s'agissait d'un effet géométrique. La forme, la position et l'inclinaison de la surface focale, qui ont été ajustées théoriquement, sont vraisemblablement quelque peu différentes de celles de la focale réelle de l'appareil. La correction à apporter aux 
valeurs de $S_{\mathrm{M}}$ sera, elle aussi, linéaire et de la forme : $S$ (corrigé) $=a \times S_{\mathrm{M}}+b$. A chaque valeur $S$ (corrigée) correspond une valeur du rayon de courbure $r$ (corrigé) et une valeur de l'induction mesurée $\left(B_{\mathbf{M}}\right)$.

D'autre part, le rapport $\frac{(B R) \text { du groupe considéré }}{r \text { (corrigé) }}$

donne une valeur théorique de l'induction $B_{\mathrm{Th}}$. La valeur $\Delta B=B_{\mathrm{Th}}-B_{\mathrm{M}}$ correspond à la correction que nous devons appliquer à la mesure de $B$. Il faut considérer, en effet, que cette mesure est effectuée en un point et ne peut donner qu'une indication sur la valeur moyenne de l'induction qui intervient dans l'expression $B R=m v / Z e$.

Les paramètres $a$ et $b$ de la correction sur $S$ ont été ajustés de façon à : $i$ ) obtenir des valeurs $\Delta B$ voisines pour les différents groupes analysés avec la même induction $B$ et focalisés en différents points de la focale; ii) obtenir une précision relative telle que :

$$
\left|\frac{\left(B_{\mathrm{M}}+B\right) r_{\text {corrigé }}-(B R)_{\text {théorique }}}{(B R)_{\text {théorique }}}\right| \leqslant 1,2 \times 10^{-4}
$$

soit une précision en énergie telle que :

$$
\left|\frac{\Delta E}{E}\right| \leqslant 2,5 \times 10^{-4} \text {. }
$$

Nota. - Cette dernière valeur est en effet compatible avec la résolution intrinsèque de l'appareil; à l'angle solide d'entrée maximal, la résolution garantie est de $\Delta E / E=1 \%$; en réduisant l'angle solide, il nous a été possible d'atteindre des résolutions en énergie de l'ordre de $5 \times 10^{-4}$. Dans ces conditions, la précision sur la position d'un pic est égale à $\pm 2,5 \times 10^{-4}$ et la précision de l'étalonnage doit être du même ordre de grandeur.

Les corrections choisies sont les suivantes :

$S_{\text {corrigé }}(\mathrm{mm})=1,0033 \times S_{\mathrm{M}}-1,700$ pour $S_{\mathrm{M}}<650$

$S_{\text {corrigé }}(\mathrm{mm})=1,001 \times S_{\mathrm{M}}-0,200$ pour $S_{\mathrm{MI}}>650$.

Un tableau (non reproduit ici) donne les valeurs $\Delta B$ en fonction de $B_{\mathrm{M}}$ telles que : $B_{\text {corrigé }}=B_{\mathrm{M}}+\Delta B$. Avec ces corrections, la figure 2 montre que les écarts

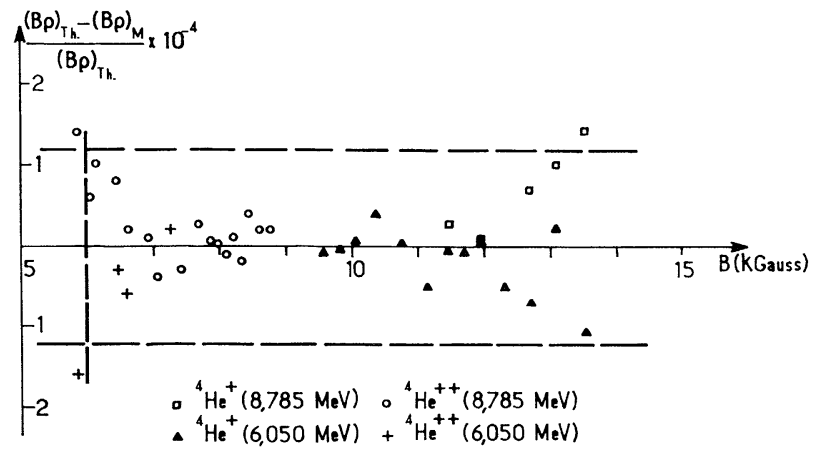

FIG. 2. - Écarts relatifs

des rigidités magnétiques vraies et mesurées.

relatifs entre les valeurs mesurées et théoriques des rigidités magnétiques restent inférieurs à $1,2 \times 10^{-4}$ dans un domaine d'induction compris entre 6 et $13 \mathrm{kG}$. 